\title{
Evaluation of Targeted Image-Guided Radiation Therapy Treatment Planning System by Use of American Association of Physicists in Medicine Task Group-119 Test Cases
}

\begin{abstract}
Background: This study aimed to evaluate the overall accuracy of the beam commissioning criteria of targeted image-guided radiation therapy (TiGRT) treatment planning system (TPS) based on the American Association of Physicists in Medicine (AAPM) Task Group Report 119 (TG-119). Methods: The work was performed using $6 \mathrm{MV}$ energy LINAC with a variable dose rate of $200 \mathrm{MU} / \mathrm{min}$ which equipped with the high-quality external TiGRT dynamic multileaf collimator model $\mathrm{H}$. The AAPM TG-119 intensity-modulated radiation therapy (IMRT) commissioning tests are composed of two preliminary tests and four clinical test cases. The clinical tests consisted of mock prostate, mock head and neck, C-shaped target, and multitarget. EDR2 film was used for evaluating the IMRT plans and point dose measured by a Pinpoint chamber positioned in slab phantom. The film analysis was done with the Sun Nuclear Corporation patient software. The dose prescription for each fraction was $200 \mathrm{cGy}$ in mock prostate, mock head and neck, C-shaped target, and multitarget. Dose distributions were analyzed using gamma criteria of $3 \%$ and $2 \%$ dose difference (DD) and 3 and $2 \mathrm{~mm}$ distance to agreement. Results: In all test cases, the gamma criteria for $2 \% / 2$ and $3 \% / 3$ were found to be $94 \%$ and $98 \%$, respectively. Results showed that the average gamma criteria result was in the range of $99.1 \%$ to $93 \%(3 \% / 3,2 \% / 2)$ overall test cases. Conclusions: Findings were favorable and in some tests were comparable with the other studies. The dose point values were within the mean values of the range reported by TG-119. Overall, the TiGRT TPS is needed to apply IMRT technique in radiation therapy centers.
\end{abstract}

Keywords: American Association of Physicists in Medicine Task Group Report 119, intensity modulated radiation therapy, radiation therapy, targeted image-guided radiation therapy, treatment planning system

\section{Introduction}

Intensity-modulated radiation therapy (IMRT) is the delivery of radiation dose to the patient through fields that have nonuniform radiation fluence. ${ }^{[1]}$ Introduction of IMRT creates the possibility of generating dramatically improved dose distributions that could be tailored to fit complex shapes. The IMRT is a promising treatment technique having advantage of delivering highly conformal dose distribution to the target volume and sparing of organs at risk. ${ }^{[2]}$ However, stringent quality assurance program is required to be instituted in dose calculation, dose delivery, and measurement system for effective and safe clinical implementation of this technique. ${ }^{[3]}$

There are many advantages of IMRT over three-dimensional radiotherapy. ${ }^{[3,4]}$ IMRT technique can be done by the use of a static multileaf collimator (MLC) and a

This is an open access journal, and articles are distributed under the terms of the Creative Commons Attribution-NonCommercialShareAlike 4.0 License, which allows others to remix, tweak, and build upon the work non-commercially, as long as appropriate credit is given and the new creations are licensed under the identical terms.

For reprints contact: reprints@medknow.com dynamic MLC technique. ${ }^{[5]}$ Task Group-119 (TG-119) IMRT commissioning tests of The American Association of Physicists in Medicine (AAPM) have been given to check precision of IMRT. It has four levels of computed tomography (CT) scan with structures named multitarget, mock prostate, mock head and neck, and C-shape. The first object of these test cases was to be able to check the whole precision of IMRT commissioning. These test cases were implemented to check the efficiency of the targeted image-guided radiation therapy (TiGRT) treatment planning system (TPS). Initial check was done to remove the effect of daily output variation of the linear accelerator. ${ }^{[5,6]}$ The beam design for planning, given dose, specific plan evaluation, and measurement of dose delivery techniques were explained in AAPM TG-119..$^{[5-7]}$ Recently, researchers have done TG-119 protocol on different

How to cite this article: Saeb M, Shahbazi-Gahrouei D, Monadi S. Evaluation of targeted image-guided radiation therapy treatment planning system by use of American Association of Physicists in Medicine task group-119 test cases. J Med Sign Sens 2018;8:95-100.

\section{Mohsen Saeb, Daryoush Shahbazi- Gahrouei, Shahram Monadi}

Department of Medical Physics, School of Medicine, Isfahan University of Medical Sciences, Isfahan, Iran

Address for correspondence: Prof. Daryoush Shahbazi-Gahrouei, Department of Medical Physics, School of Medicine, Isfahan University of Medical Sciences, Isfahan, Iran.

E-mail: shahbazi@med.mui.ac.ir

Website: www.jmss.mui.ac.ir DOI: 10.4103/jmss.JMSS_44_17 
planning systems, different detectors, and LINAC machines with different photon energies and obtained different gamma criteria. ${ }^{[8-16]}$

According to the author's best knowledge, there is a lack of commissioning studies verifying TPS and delivered accurate dose in IMRT using TiGRT TPS for $6 \mathrm{MV}$ photon energy LINAC. Furthermore, there is no report on the application of TG-119 test on TiGRT TPS with Primus accelerator machine and TiGRT dynamic MLC (DMLC) system. In this work, TG-119 test on TiGRT TPS was done with Primus accelerator system and TiGRT DMLC. This study aimed to evaluate the overall accuracy of the beam commissioning criteria of TiGRT TPS based on the TG-119 guidelines.

\section{Materials and Methods}

This work was performed using $6 \mathrm{MV}$ photon beam energy linear accelerator (Siemens Primus, USA) and the variable dose rate of $200 \mathrm{MU} / \mathrm{min}$. It was equipped with high-quality external TiGRT DMLC $\mathrm{H}$ with 51 pairs of MLCs with 0.6 and $0.36 \mathrm{~cm}$ resolution in isocenter. The maximum length of field size is $32 \mathrm{~cm}$ and the MLC leaf length is $26.54 \mathrm{~cm}$. In this MLC, maximum over travel from the center is $12.8 \mathrm{~cm}$ and minimum gap between leaf pairs is $0.19 \mathrm{~cm}$. The major leaf speed is $2.97 \mathrm{~cm} / \mathrm{s}$. The phantom was water-equivalent plastic phantom with a density of $1.03 \mathrm{~g} / \mathrm{cm}^{3}$ and dimensions of $30 \mathrm{~cm} \times 30 \mathrm{~cm} \times 15 \mathrm{~cm}$ that is typically squares or rectangles. This phantom has a suitable thickness to put the ionization chamber at its center for the aim of dosimetric measurements. Another advantage of this phantom is permitting planar dose measurement (kinds of film) to be done on coronal planes. CT images of this phantom were obtained and then imported in the TPS. During CT scan, some markers were put in the phantom for performing the best contouring and also some points were defined to calculate dose in the TPS. TiGRT TPS was calculated with the full scatter convolution algorithm. The system is able to carry conformal and IMRT techniques. The system is able to perform forward and reverse calculations. TiGRT TPS supports step and shoot and sliding IMRT technique. Pinpoint chamber (PTW, Freiburg, Germany, model 31014) with the effective or sensitive volume of $0.015 \mathrm{~cm}^{3}$ was used to point dose measurements.

The AAPM TG-119 IMRT commissioning tests are composed of two preliminary tests and four clinical test cases. $^{[5]}$ The preliminary tests are about the evaluation of the precision dose calculation, and clinical tests consist of test prostate, head and neck, C-shaped target, and multitarget ${ }^{[5]}$ which are shown in Figure 1. The test cases were done with a CT scanner (Siemens SOMATOM EMOTION, Germany) and imported into the TiGRT TPS.

Figure 1 shows two-dimensional (2D) view of test structure sets which are described as follow: Figure 1a demonstrates three cylindrical targets named multitarget cases, which were drawn along the axis of rotation, each having a diameter of $4.0 \mathrm{~cm}$ and length of $4.0 \mathrm{~cm}$ and including central target, superior target, and inferior target. Figure 1b shows the mock prostate case including the prostate, planning target volume (PTV), rectum, and bladder. The rectum was a cylinder with a diameter of $1.5 \mathrm{~cm}$. One-third of the rectum volume was inside the PTV of the prostate. The bladder was roughly ellipsoidal and was centered on the superior portion of the prostate. The prostate clinical target volume (CTV) is roughly ellipsoidal with right lateral (RL), anterior-posterior (AP), and superior interior (SI) dimensions of 4.0, 2.6, and $6.5 \mathrm{~cm}$, respectively. The prostate PTV is expanded to $0.6 \mathrm{~cm}$ around the CTV. The rectum is a cylinder with a diameter of $1.5 \mathrm{~cm}$ that abuts the indented posterior aspect of the prostate. The PTV includes about one-third of the rectal volume on the widest PTV slice. The bladder is roughly ellipsoidal with RL, AP, and SI dimensions of 5.0, 4.0, and $5.0 \mathrm{~cm}$, respectively, and is centered on the superior aspect of the prostate.

Figure 1c illustrated the mock head and neck case including the PTV, spinal cord, right parotid, and left parotid. The PTV is retracted from the skin by $0.6 \mathrm{~cm}$. There is a gap of about $1.5 \mathrm{~cm}$ between the cord and the PTV.

Figure 1d shows the C-shape case including a C-shape target that surrounded a central isolation structure called core. The center core is a cylinder with $1 \mathrm{~cm}$ in radius. The interval between the core and the PTV is $0.5 \mathrm{~cm}$; therefore, the interior curve of the PTV is $1.5 \mathrm{~cm}$ in radius. The outer curve of the PTV is $3.7 \mathrm{~cm}$ in radius. The PTV and core are 8 and $10 \mathrm{~cm}$ long, respectively. The frontal view of the treatment planning software is shown in Figure 2.

According to AAPM TG-119, each experiment was done with film to be placed in at least on coronal plane and to

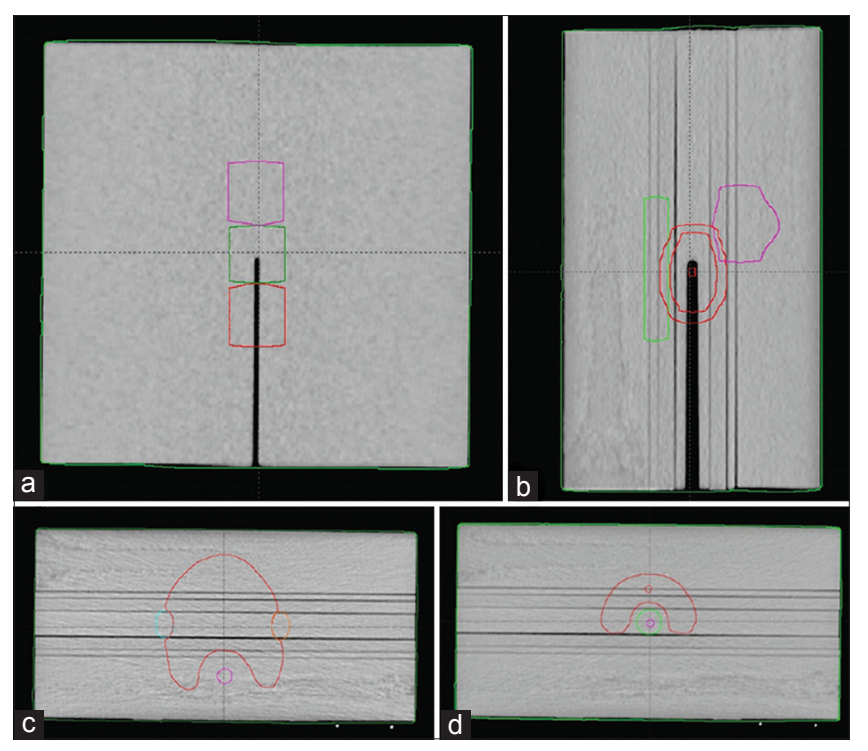

Figure 1: Two-dimensional view of test structure sets: (a) Multitarget, (b) prostate, (c) head and neck and (d) C-shape 




Figure 2: The frontal view of the treatment planning algorithms

be exposed to all fields of the phantom with the planned collimator and gantry angles. ${ }^{[9]}$ The entire center tries to gain the best result for film dosimetry. ${ }^{[10]}$ Dose distributions were analyzed applying gamma criteria of 3\% and $2 \%$ DD and 3 and $2 \mathrm{~mm}$ distance to agreement (DTA). The planar dose distributions obtained with the film could be normalized to the dose measured with the chamber at an appropriate point in a high-dose and low-gradient region.

EDR2 (Kodack, Japan) film has appropriate spatial resolution and is independent of beam angle, energy, and dose rate. ${ }^{[11]}$ Film dosimeters have some advantages for being 2D detectors that give permanent records of the measuring dose distribution at high resolutions. ${ }^{[11,12]}$

EDR2 film is a very slow speed and fine-grain film. Double emulsion active layers, formed by very fine monodispersed cubic microcrystals, are coated on a $0.18 \mathrm{~mm}$ Easter base, which allows processing of film in a conventional rapid film processor. ${ }^{[17]}$ The same batch of $10 \times 12$ inch EDR2 ready pack film and its calibration files were used throughout this study. A Kodak Xomat film processor was used for processing irradiated EDR2 films. ${ }^{[12]}$

A calibration data set for EDR2 film type was acquired by placing a film under a $1.5 \mathrm{~cm}$ thickness solid water phantom with $20 \mathrm{~cm}$ solid water underneath. The dose output to the water from linear accelerator was calibrated using an ion chamber calibrated by the Accredited Dosimetry Calibration Laboratory (ADCL).

Films were irradiated by $6 \mathrm{MV}$ photon beams perpendicular to the beam axis, with a field size of $5 \mathrm{~cm} \times 5 \mathrm{~cm}$. The dose delivered at a given depth was calculated by multiplying the delivered MUs and output factor for $5 \mathrm{~cm} \times 5 \mathrm{~cm}$ field size. Care was taken to minimize the contribution of collimator scattered photons reaching the unirradiated region of the film. Fourteen dose points were obtained using four films by irradiating the films with different doses up to $700 \mathrm{cGy}$. The perpendicular irradiation geometry was chosen for the film calibration because IMRT plans studied would use either coplanar or noncoplanar beam arrangement; therefore, neither perpendicular nor parallel irradiation setup for calibration would be corrected for the direction of film exposure and cannot be corrected by the calibration process. The processed films were scanned, and the region of interest (ROI) of $4 \mathrm{~mm} \times 4 \mathrm{~mm}$ was selected at the middle of the irradiated region to get the optical density (OD) measurement. For dose range of 0-700 cGy, EDR2 films responded with a linear response up to $500 \mathrm{cGy}$ in the OD range of $0.20-3.04 .^{[12]}$

In this study, as it will be mentioned in the following parts, it is possible to have both film and chamber on the central measurement plane so that the film response can be normalized to the chamber. The used phantom was scanned for planning and measurements goal. The plans were done on the phantom with the structures outlined on it. The chamber measurements were to be made with all fields irradiating the phantom using the planned gantry and collimator angles. For most of the tests, measurements were made in at least two locations, one in the target and another in a low-dose avoidance structure. The doses were expected to be at least $30 \mathrm{cGy}$, so issues with very low-dose measurements would not arise. Conversion of chamber reading to dose was done by first irradiating the phantom with parallel-opposed $10 \mathrm{~cm} \times 10 \mathrm{~cm}$ fields arranged isocentrically and establishing the ratio of reading to planned dose in that geometry. This was done to reduce the effects of daily LINAC output variations and differences between the phantom and liquid water.

Each test called for a film to be placed in at least one coronal plane and to be exposed to all fields irradiating the phantom with the planned gantry and collimator angles. 
Dose distributions were analyzed using gamma criteria of $3 \%$ and $2 \%$ DD and 3 and $2 \mathrm{~mm}$ DTA. The planar dose distributions obtained with the film could be normalized to the dose measured with the chamber at a suitable point in a high-dose and low-gradient region. The film analysis was done with the SNC patient software (Sun Nuclear Corporation, Melbourne, FL, USA).

Two preliminary tests with simple fields irradiating the phantom were requested to demonstrate the reliability of the assessment system for non-IMRT dose delivery, followed by five tests of IMRT plans with increasing complexity.

Preliminary tests were done on slab phantom. Preliminary tests included two examinations. Test 1 included parallel opposed AP: PA $10 \mathrm{~cm} \times 10 \mathrm{~cm}$ fields to deliver $200 \mathrm{cGy}$ at the isocenter. Test 2 included a set of five AP: PA bands of $3 \mathrm{~cm}$ using asymmetric jaws, with doses ranging from 40 to 200 cGy which could be done using asymmetric jaws. The central dose was measured with chamber and the dose distribution on the central plane was measured by film.

For test cases, beam arrangements for sliding IMRT were done as mentioned in AAPM TG-119. For multitarget and mock prostate cases, seven static beams were placed at $50^{\circ}$ intervals. For mock head and neck and C-shape cases, nine static beams were placed at $40^{\circ}$ intervals. All plans were made according to the goal described in TG-119. The isodose and dose-volume histogram were used as the evaluation tool. As per our institutional policy, for patient quality assurance, the plans were transferred to the QA map-check phantom in TiGRT TPS with the original gantry angle and collimator angle at $0^{\circ}$ for its verification.

Data analysis

Point doses were measured with the pinpoint (PTW, Freiburg, Germany, model 31014) chamber and compared to point doses calculated by the TPS, which were taken as the mean dose for a $0.125 \mathrm{~cm}^{3}$ ROI centered around the mid-chamber position in the planning CT image set. For each point dose, a percentage difference was computed using the following formula: ${ }^{[13]}$

$$
\operatorname{diff}=\frac{D_{\text {measured }}-D_{\text {calc }}}{D_{\text {prescrip }}} 100(\%)
$$

Where $D_{\text {measured }}$ is the measure dose, $D_{\text {calc }}$ is the calculated dose, and $D_{\text {prescrip }}$ is the prescribed dose. EDR2 films measurements were compared to planar doses calculated by the TPS at a dose grid resolution of $2 \mathrm{~mm} \times 2 \mathrm{~mm}$. Measured and calculated planar doses were compared using gamma analysis using a 2\% DD and distance to agreement of $2 \mathrm{~mm}$. The analysis was done for $3 \%$ and $3 \mathrm{~mm}$ as well.

\section{Results}

For pretest 1, the measured and calculated point dose at isocenter is given in Table 1.

Experimental film dosimetry resulted in passing gamma criteria of $3 \%$ DD and $3 \mathrm{~mm}$ DTA with accuracy of $97 \%$. Pretest 2 contained a set of five AP: PA bands of $3 \mathrm{~cm}$ using asymmetric jaws, with doses ranging from 40 to 200 cGy. Figure 3 shows the dose distribution for preliminary tests and Table 2 shows the results of this measurement.

Table 3 showed the results for the test cases, multitarget, prostate, head and neck, and C-shape.

The differences between measured doses by ion chamber and that calculated using TiGRT TPS should be within $3 \%$ for whole plan in all location sites (multitarget, mock prostate, mock head and neck, and C-shape). If the result gives a variation higher than $4 \%$, the QA procedure is repeated. Table 3 shows that $100 \%$ of the measurements vary $<3 \%$ from calculated dose. This table also shows the difference errors of the percentage at each point dose measurement site.

EDR2 film dosimetry was used to compare the calculated and measured doses. Two separated gamma criteria DD/DTA for the entire location site are compared: $2 \% / 2 \mathrm{~mm}$ and $3 \% / 3 \mathrm{~mm}$. The DDs between measured dose (LINAC delivery) and calculated dose (TPS) are evaluated.

As can be seen from Table 4 , the criteria of $2 \%$ DD and $2 \mathrm{~mm}$ DTA show that the gamma passed rates are $>90.7 \%$. On the other hand, the criteria of $3 \%$ DD and $3 \mathrm{~mm}$ DTA

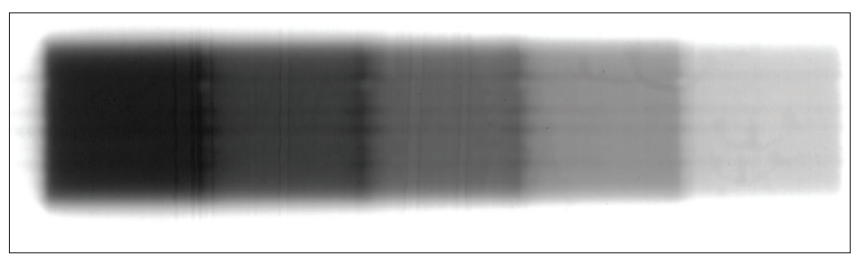

Figure 3: Dose distribution for pretest

Table 1: Results of point dose measurement in the phantom for pretest 1

\begin{tabular}{llcccc}
\hline Test name & Position the chamber & Delivery dose (cGy) & Planning calculated dose (cGy) & Measured dose (cGy) & Difference (\%) \\
\hline Pretest AP/PA & Phantom midline & 200 & 200 & 200.2
\end{tabular}
$\mathrm{AP}$ - Anterior posterior; PA - Posterior anterior

Table 2: Results of point dose measurement in the phantom for pretest 2

\begin{tabular}{llcccc}
\hline Test name & Position the chamber & Delivery dose (cGy) & Planning calculated dose (cGy) & Measured dose (cGy) & Difference (\%) \\
\hline Pretest AP/PA & Phantom midline & 200 & 200 & 200.1 & 0.5
\end{tabular}


Table 3: Result of point dose measurement in phantom for multitarget, mock prostate, mock head and neck, and C-shaped

\begin{tabular}{|c|c|c|c|c|}
\hline Location site & Measurement point & Calculated dose (cGy) & Measured dose (cGy) & Difference (\%) \\
\hline \multirow[t]{3}{*}{ Multi-target } & Central target & 186.11 & 183.32 & -1.4 \\
\hline & Superior target & 133.16 & 136.07 & 1.46 \\
\hline & Inferior target & 95.20 & 97.95 & 1.38 \\
\hline \multirow{2}{*}{$\begin{array}{l}\text { Mock } \\
\text { prostate }\end{array}$} & Isocenter, in the mid-PTV & 174.95 & 175.22 & 0.14 \\
\hline & $2.5 \mathrm{~cm}$ posterior, midrectum & 145.24 & 149.05 & 1.90 \\
\hline \multirow{2}{*}{$\begin{array}{l}\text { Mock head/ } \\
\text { neck }\end{array}$} & Isocenter, in the mid-PTV & 220.65 & 224.29 & 1.82 \\
\hline & $4 \mathrm{~cm}$ posterior mid spinal cord & 134.09 & 130.51 & -1.79 \\
\hline \multirow[t]{2}{*}{ C-shape } & Central core & 88.15 & 90.87 & 1.36 \\
\hline & Mid-PTV, $2.5 \mathrm{~cm}$ anterior to isocenter & 200.42 & 197.14 & -1.64 \\
\hline
\end{tabular}

PTV - Planning target volume

Table 4: Results of gamma analysis of all location sites

for dynamic intensity-modulated radiation therapy

\begin{tabular}{|c|c|c|c|}
\hline Location site & $\begin{array}{l}\text { Number of the } \\
\text { beam }\end{array}$ & $\begin{array}{c}\text { Gamma pass } \\
\text { rate with } 3 \% \\
\text { and } 3 \mathrm{~mm}\end{array}$ & $\begin{array}{c}\text { Gamma pass } \\
\text { rate with } 2 \% \\
\text { and } 2 \mathrm{~mm}\end{array}$ \\
\hline Multitarget & 7 with $50^{\circ}$ interval & 99.1 & 96.6 \\
\hline Mock prostate & 7 with $50^{\circ}$ interval & 96.9 & 90.7 \\
\hline $\begin{array}{l}\text { Mock head and } \\
\text { neck }\end{array}$ & 9 with $40^{\circ}$ interval & 98.2 & 94.1 \\
\hline C-shape & 9 with $40^{\circ}$ interval & 97.7 & 93.4 \\
\hline
\end{tabular}

showed that gamma passed rates are $>96.9 \%$. The best results were obtained for multitarget location site.

\section{Discussion}

Using TG-119 protocol to verify the accuracy of measurements and compliance with the calculation TPS, it will be claimed to use treatment planning software with more confidence in IMRT techniques.

TG-119 protocol was applied to evaluate the accuracy and precision of the TiGRT TPS. TiGRT TPS performs the appropriate and admirable inverse plan for the sliding (dynamic) IMRT.

As shown in Table 4, results of this work for multitarget test were acceptable and had more confidence than other studies such as Ezzel et al. and Mynampat et al..$^{[5,7]}$

Comparing sliding IMRT technique with the step and shoot IMRT technique accompanying using the TG-119 criterion showed that the sliding IMRT technique has true measurements and has fewer faults. ${ }^{[14,17]}$ In this work, small ionization chamber (Pinpoint) was used instead of larger ionization chamber (Farmer) which was used by other researchers. ${ }^{[14-16]}$ The main advantage of this small chamber is its use for increasing the accuracy, in particular, in high-dose gradient and edge of the fields. ${ }^{[18]}$

Comparing the results with other relevant studies, film dosimetry can be used instead of 2D arrays. Film has more resolution than the other $2 \mathrm{D}$ arrays such as Octavius and Mapcheck2. ${ }^{[9]}$
By comparing point of view of planning cost and simplicity with other machines, ${ }^{[5,7]}$ it is important to notice that Primus LINAC which added on TiGRT DMLC is cheaper than other complex LINAC machines.

The data from this study were more accurate than other studies in some tests such as multitarget. ${ }^{[5,7]}$ Fortunately, the results of this work regarding the dose point were within the mean values of the range reported by TG-119. [5]

The main advantage of this study is its clinical application. One of the strengths of this study was using film dosimetry instead of the $2 \mathrm{D}$ which has a higher resolution than the $2 \mathrm{D}$ arrays. ${ }^{[12]}$ The result of gamma pass rate is a good reason to use TiGRT TPS for IMRT techniques.

\section{Conclusions}

Dose distribution was in the range of $99.1 \%$ to $93 \%$ using gamma criteria of $3 \%$ and $2 \%$ DD and 3 and $2 \mathrm{~mm}$ distance to agreement $(3 \% / 3$ and $2 \% / 2)$, respectively overall test cases. Findings of this work were very favorable and in some tests were comparable with other studies. Furthermore, the results of dose point were within the mean values of the range reported by TG-119. Overall, the TiGRT TPS may be suitable for use in IMRT DMLC radiation therapy techniques.

Acknowledgment

The authors thank all of the technicians for providing us technical support required for carrying out this work.

Financial support and sponsorship

This work is a part of $\mathrm{PhD}$ thesis which was financially supported (Grant No. 394597) by Isfahan University of Medical Sciences.

\section{Conflicts of interest}

There are no conflicts of interest.

\section{References}

1. Webb S. The physical basis of IMRT and inverse planning. Br J 
Radiol 2003;76:678-89.

2. Rezaee V, Shahbazi-Gahrouei D, Monadi S, Saeb M. Evaluation of error doses of treatment planning software using solid anthropomorphic phantom. J Isfahan Med Sch 2016;34:908-13.

3. Karbalaee M, Shahbazi-Gahrouei D, Tavakoli MB. An approach in radiation therapy treatment planning: A Fast, GPU-based Monte Carlo method. J Med Signals Sens 2017;7:108-13.

4. Shahbazi-Gahrouei D, Saeb M, Monadi S, Jabbari I. Clinical implications of TiGRT algorithm for external audit in radiation oncology. Adv Biomed Res 2017;6:117.

5. Ezzell GA, Burmeister JW, Dogan N, LoSasso TJ, Mechalakos JG, Mihailidis D, et al. IMRT commissioning: Multiple institution planning and dosimetry comparisons, a report from AAPM task group 119. Med Phys 2009;36:5359-73.

6. Galvin JM, Ezzell G, Eisbrauch A, Yu C, Butler B, Xiao Y, et al. Implementing IMRT in clinical practice: A joint document of the American society for therapeutic radiology and oncology and the American association of physicists in medicine. Int J Radiat Oncol Biol Phys 2004;58:1616-34.

7. Mynampati DK, Yaparpalvi R, Hong L, Kuo HC, Mah D. Application of AAPM TG 119 to volumetric arc therapy (VMAT). J Appl Clin Med Phys 2012;13:3382.

8. Clemente-Gutiérrez F, Pérez-Vara C. Dosimetric validation and clinical implementation of two $3 \mathrm{D}$ dose verification systems for quality assurance in volumetric-modulated arc therapy techniques. J Appl Clin Med Phys 2015;16:5190.

9. Wen N, Zhao B, Kim J, Chin-Snyder K, Bellon M, Glide-Hurst C, et al. IMRT and RapidArc commissioning of a TrueBeam linear accelerator using TG-119 protocol cases. J Appl Clin Med Phys 2014;15:4843.
10. Keivan H, Shahbazi-Gahrouei D, Shanei A. Assessment of imprecise small photon beam modeling by two TPS algorithms. J Med Signals Sens, 2018;8:39-45.

11. Casanova Borca V, Pasquino M, Russo G, Grosso P, Cante D, Sciacero $\mathrm{P}$, et al. Dosimetric characterization and use of GAFCHROMIC EBT3 film for IMRT dose verification. J Appl Clin Med Phys 2013;14:4111.

12. Sankar A, Ayyangar KM, Nehru RM, Kurup PG, Murali V, Enke CA, et al. Comparison of kodak EDR2 and gafchromic EBT film for intensity-modulated radiation therapy dose distribution verification. Med Dosim 2006;31:273-82.

13. Dumitrache M, Tănase A. Gamma dose distribution evaluation of $\mathrm{XiO}$ treatment planning system for static field IMRT, using AAPM TG-119. Rom Rep Phys 2016;68:1130-9.

14. McVicker D, Yin FF, Adamson JD. On the sensitivity of TG-119 and IROC credentialing to TPS commissioning errors. J Appl Clin Med Phys 2016;17:34-48.

15. Nithya L, Raj NA, Rathinamuthu S, Pandey MB. Analyzing the performance of the planning system by use of AAPM TG 119 test cases. Radiol Phys Technol 2016;9:22-9.

16. Kadam A, Sharma SD. Estimation of local confidence limit for $6 \mathrm{MV}$ photon beam IMRT system using AAPM TG 119 test protocol. Int Cancer Ther Oncol 2016;4:4110.

17. Haus AG, Dickerson RE, Huff KE, Monte S, Schlager BA, Atanas $\mathrm{M}$, et al. Evaluation of a cassette-screen-film combination for radiation therapy portal localization imaging with improved contrast. Med Phys 1997;24:1605-8.

18. Jomehzadeh A, Shahbazi-Gahrouei D, Jahanbakhsh V. Effect of material and wall thickness buildup caps on the head scatter factor measurements in irregular fields shielded by cerrobend. J Med Signals Sens 2017;7:247-51.

\section{BIOGRAPHIES}

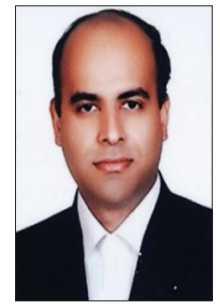

Mohsen Saeb received his BSc degree in Physics from Isfahan University, Iran, 1999. He obtained MSc and PhD degrees from the department of Medical Physics of Isfahan University of Medical Sciences, Isfahan, Iran, in 2003 and 2018, respectively. His research interest is radiation dosimetry and the Physics of radiation Therapy.

Email: mohsen.saeb93@gmail.com

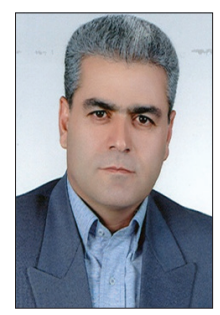

Daryoush Shahbazi-Gahrouei Professor of Medical Physics, BSc in Physics (School of Science, Isfahan University, Iran, 1987), MSc in Medical Physics (Tarbiat Modarres University, Tehran, Iran, 1991), PhD in Medical Physics (University of Western Sydney and St. George Cancer Care Centre, Sydney, Australia, 2000).

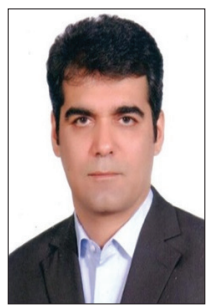

Shahram Monadi received his BSc degree in Physics from Isfahan University, Isfahan, Iran, 1990 and MSc of Medical Physics from the department of Medical Physics at Tehran University of Medical Sciences, Tehran, Iran, in 2001. He is head of Physicists for radiation therapy in Seyed Al-Shohada hospital, Isfahan, Iran.

Email: monadi46@gmail.com

Email: shahbazi@med.mui.ac.ir 